\title{
Effect of Environmental Temperature and Humidity on Permethrin Biomarkers of Exposure in U.S. Soldiers Wearing Permethrin-Treated Uniforms
}

\author{
Alexis L. Maule, ${ }^{1,2}$ Kristin J. Heaton, ${ }^{1,3}$ Bruce Cadarette, ${ }^{4}$ Kathryn M. Taylor, ${ }^{1}$ Katelyn I. Guerriere, ${ }^{1}$ Caitlin C. Haven, ${ }^{1}$ \\ Matthew M. Scarpaci, ${ }^{1,2}$ Robert W. Kenefick, ${ }^{4}$ Maria Ospina, ${ }^{5}$ Antonia M. Calafat, ${ }^{5}$ and Susan P. Proctor ${ }^{1,2,6 *}$ \\ ${ }^{1}$ United States Army Research Institute of Environmental Medicine, Military Performance Division, Natick, Massashusetts; ${ }^{2}$ Henry M. Jackson \\ Foundation for the Advancement of Military Medicine, Bethesda, Maryland; ${ }^{3}$ Boston University School of Public Health, Department of \\ Environmental Health, Boston, Massachusetts; ${ }^{4}$ United States Army Research Institute of Environmental Medicine, Thermal and Mountain \\ Medicine Division, Natick, Massachusetts; ${ }^{5}$ Centers for Disease Control and Prevention, National Center for Environmental Health, Division of \\ Laboratory Sciences, Atlanta, Georgia; ${ }^{6}$ VA Boston Healthcare System, Research Service, Boston, Massachusetts
}

\begin{abstract}
Environmental factors, including high temperature and humidity, can influence dermal absorption of chemicals. Soldiers can be dermally exposed to permethrin while wearing permethrin-treated uniforms. This study aimed at examining the effects of high temperature and a combined high temperature and humid environment on permethrin absorption compared with ambient conditions when wearing a permethrin-treated uniform. Twenty-seven male enlisted soldiers wore study-issued permethrin-treated army uniforms for 33 consecutive hours in three different environments: 1 ) simulated high temperature $\left(35^{\circ} \mathrm{C}, 40 \%\right.$ relative humidity [rh] $\left.)(n=10), 2\right)$ simulated high temperature and humidity $\left(30^{\circ} \mathrm{C}\right.$, $70 \%$ rh) $(n=10)$, and 3$)$ ambient conditions $\left(13^{\circ} \mathrm{C}, 60 \% \mathrm{rh}\right)(n=7)$. Spot urine samples, collected at 21 scheduled time points before, during, and after wearing the study uniforms, were analyzed for permethrin exposure biomarkers (3phenoxybenzoic acid, cis- and trans-3-(2,2-dichlorovinyl)-2,2-dimethylcyclopropane-1-carboxylic acid) and creatinine. Biomarker concentrations were $60-90 \%$ higher in the heat and combined heat/humidity groups $(P<0.001-0.022)$ than the ambient group. Also, the average daily permethrin dose, calculated 12 hours after removing the treated uniforms, was significantly higher in the heat $(P=0.01)$ and the heat/humidity $(P=0.03)$ groups than the ambient group. There were no significant differences in biomarker concentrations or computed average daily dose between the heat and the heat/ humidity groups. Both hot and combined hot and humid environmental conditions significantly increased permethrin absorption in soldiers wearing permethrin-treated uniforms.
\end{abstract}

\section{INTRODUCTION}

Since 2013, all U.S. Army personnel have been required to wear uniforms that have been factory-treated, post-tailored with permethrin, regardless of military occupational specialty (MOS), duty location, or deployment status. ${ }^{1}$ This uniform regulation was implemented to protect soldiers from biting insects and vector-borne diseases in a variety of training and deployment environments. Each military service requires permethrin-treated clothing to meet Environmental Protection Agency (EPA) and Federal Insecticide, Fungicide, and Rodenticide Act standards, along with their own service-specific permethrin-treated fabric levels and efficacy (\% bite) protection requirements. The U.S. Army requires all new, unwashed uniform permethrin concentrations to be within a minimum of $0.095 \mathrm{mg} / \mathrm{cm}^{2}$ and maximum of $0.135 \mathrm{mg} / \mathrm{cm}^{2}$.

Permethrin acts as both an insect repellant and an insecticide. ${ }^{2}$ In field studies of outdoor workers and military personnel, permethrin-treated uniforms demonstrated high efficacy against mosquito ${ }^{3,4}$ and tick bites. ${ }^{5-7}$ For example, soldiers wearing permethrin-treated uniforms in French Guiana, an area of high malaria transmission, had no new cases of malaria over the study period, compared with 11 new cases among 125 soldiers wearing conventional uniforms. ${ }^{4}$ Also, a significant reduction in tick bites was observed during the training session where soldiers wore permethrin-treated uniforms compared with an equivalent training period when soldiers wore untreated uniforms. ${ }^{5}$

*Address correspondence to Susan P. Proctor, Military Performance Division, United States Army Research Institute of Environmental Medicine, 10 Gen Greene Ave., Bldg. 42, Natick, MA 01760-5007. E-mail: susan.p.proctor.civ@mail.mil
There is limited health risk information related to the unacceptable levels of exposure to permethrin through the dermal route. From other human studies reported to date, ${ }^{8,9}$ biomarker concentrations involving wear of treated clothing are 100-200 times higher than U.S. general population reference range concentrations. ${ }^{10}$ However, on average, the estimated dose through the dermal route is lower than the WHO acceptable daily intake (ADl; $50 \mu \mathrm{g} / \mathrm{kg} /$ day) for oral ingestion, assuming $50 \%$ absorption of oral dose (or $25 \mu \mathrm{g} / \mathrm{kg} /$ day). There are limited reports of acute human health impacts related to the increased number of people wearing permethrin-treated clothing presumably because of permethrin's demonstrated low mammalian toxicity when absorbed dermally. ${ }^{11}$ However, the potential for longer term health issues does exist. Permethrin has been classified by the U.S. EPA as a potential carcinogen because there exists some evidence to suggest permethrin may cause cancer in animals.

Biomonitoring studies have shown that individuals wearing permethrin-treated clothing have significantly higher concentrations of urinary permethrin metabolites than those wearing untreated or conventional clothing, suggesting dermal exposure to the active ingredient as a result of wearing treated clothing. ${ }^{12-14}$ Among German and U.S. Army soldiers, the duration of wear was associated with permethrin biomarkers measured in urine.$^{8,12,14,15}$ In addition to the length of wear time, environmental temperature, physical workload, and perspiration have been associated with increased permethrin absorption in forestry workers and U.S. Army soldiers wearing permethrin-treated clothing and uniforms, respectively. ${ }^{16-18}$ These results correspond to a body of literature that indicates absorption of chemicals following dermal exposure is affected by environmental conditions. First, temperature and humidity may impact the amount of 
permethrin that leaches from the clothing, increasing the amount of active ingredient available for transfer to the skin surface. ${ }^{4,12,19}$ Dermal absorption can also be increased by enhanced blood flow to portions of the skin, sweating, and skin hydration, which in turn are all influenced by the temperature and humidity of the environment. ${ }^{19-22}$ The skin can also act as a reservoir, especially for lipophilic chemicals, and an increase in dermal absorption may increase the chemical reservoir in the stratum corneum. ${ }^{21,22}$

The U.S. Army trains and operates in a myriad of environments, including temperate, desert (hot and dry), and tropical (hot and humid) zones. To our knowledge, there have been no studies examining the effects of combined high temperature and humid environmental conditions on permethrin exposure in military personnel wearing permethrintreated uniforms. We hypothesized that permethrin biomarkers would be higher among those exposed to high temperature and combined temperature and humidity than those exposed to ambient conditions, and we explored the potential impact of whether increased humidity would result in even higher exposures as a result of the effect of the role of increased sweating and dermal exposure. This study used simulated environmental conditions to examine the effects of high temperature and a combination of high temperature and humidity on permethrin exposure among soldiers wearing permethrin-treated uniforms over 33 hours to reflect a deployment or training scenario.

\section{METHODS}

This protocol was reviewed and approved by the U.S. Army Research Institute of Environmental Medicine Institutional Review Board (IRB) and the U.S. Army Medical Research and Materiel Command IRB. The investigators have adhered to the policies for protection of human subjects as prescribed in Army Regulation 70-25, and the research was conducted in adherence with the provisions of 32 Code of Federal Regulations (CFR) Part 219. All participants were briefed on study procedures and gave their informed consent before the research study. The involvement of the CDC laboratory did not constitute engagement in human subject research.

Study design. Twenty-seven male soldiers were recruited from a pool of human research volunteers at the Natick Soldier Systems Center (NSSC), Natick, MA, to participate in one of the three study groups. Participants were included if they did not report an allergy to permethrin or have a history of heat-related illness, although no subjects were excluded based on these criteria. All participants, regardless of the study group, wore study-issued permethrin-treated army uniforms for 33 consecutive hours to mimic sustained or continuous wear time during military operations. Participants in the heat group $(n=10)$ wore the uniforms in a simulated hot environment $\left(35^{\circ} \mathrm{C}, 40 \% \mathrm{rh}\right)$, whereas those in the heat/ humidity group ( $n=10$ ) wore the study-issued uniforms in a simulated, controlled hot and humid environment $\left(30^{\circ} \mathrm{C}\right.$, $70 \% \mathrm{rh})$. The temperature and humidity settings in the simulated environments were chosen to achieve environmental conditions that were parallel on the heat index scale but permit an examination of the additional impact of humidity. Those assigned to the final group (ambient group, $n=7$ ) wore the uniforms in uncontrolled ambient conditions (average conditions $13^{\circ} \mathrm{C}, 60 \% \mathrm{rh}$ ).

The study design and data collection procedures are outlined in detail in Proctor et al. ${ }^{18}$ In brief, all study groups completed an 11-day protocol with study activities occurring on 9 of the 11 days (Figure 1). At baseline on day 1, demographic information was collected using a written questionnaire, and height $(\mathrm{cm})$ and weight $(\mathrm{kg})$ were measured. Percent (\%) body fat was calculated using the threesite skinfold method described in Baty International ${ }^{23}$ and Proctor et al. ${ }^{18}$ On day 3 , subjects reported to the NSSC Doriot Climatic Chambers to sleep overnight in the building dormitory with study staff supervision. New permethrintreated army combat uniforms (i.e., jacket and trousers) were laundered once and then issued to soldier volunteers to wear for 33 hours, starting on the morning of day $4(\sim 0,700)$ through the afternoon of day $5(\sim 1,600)$. Subjects wore their own undergarments, socks, and boots while wearing the studyissued uniform. A subset of study-issued uniforms worn under the different environmental conditions during the study were tested for permethrin concentration ${ }^{24,25}$ at the end of the study. Also, unworn uniforms that were laundered once were tested to provide fabric concentrations at the start of the study.

After subjects were issued their study uniforms on day 4, they were given an activity monitor (Philips Actical) ${ }^{26}$ to be worn on their right ankle/calf above their boot for the 33 hours the study uniform was worn. These monitors estimated the energy expenditure (kcal) at 1-minute intervals. The 1-minute intervals were summed over the 33 hours to determine the total energy expenditure (kcal) during the time the study-issued uniform was worn.

Subjects in the heat and heat/humidity groups entered the Doriot Tropical Chambers at approximately 0730 on day 4 and exited at approximately 1600 on day 5 . The heart rate and core body temperature were monitored every 30 minutes to ensure the safety of participants. A detailed description of chamber activities is described in Proctor et al. ${ }^{18}$ In brief, bunk beds were provided for participants to sleep overnight in the Tropical Chambers. Overnight conditions for the heat group were reduced to $30^{\circ} \mathrm{C}, 50 \%$ rh to simulate cooler nighttime conditions. The heat/humidity group's overnight conditions remained the same as the daytime conditions. While in the chamber, participants in both groups completed four 1-hour ruck marches; two on day 4, and two on day 5 at scheduled time points. All groups marched on a treadmill at a rate of 2 mph; participants in the heat group wore a 55-lb weighted

\begin{tabular}{|l|l|l|l|l|l|l|l|l|l|l|l|}
\hline & Friday & Saturday & Sunday & Monday & Tuesday & Wednesday & Thursday & Friday & Saturday & Sunday & Monday \\
\hline Time $\neq$ & Day 1 & Day 2 & Day 3 & Day 4 & Day 5 & Day 6 & Day 7 & Day 8 & Day 9 & Day 10 & Day 11 \\
\hline $0600^{*}$ & U1 & & & U4* & U8* & U12* & U16* & U18* & U20* & & U21* \\
0900 & & & & U5 & U9 & U13 & & & & \\
1300 & U2 & & & U6 & U10 & U14 & U17 & U19 & & \\
1900 & & & U3 & U7 & U11 & U15 & & & & \\
\hline
\end{tabular}

FIGURE 1. ₹Collection times are approximate, urine sample collected within an hour from the given time. *First morning void. Gray-shading $=$ time under study environmental conditions wearing study-issued uniform; Black = no study activities on days 2 and 10. 
vest. Participants in the heat/humidity group wore an unweighted vest in an effort not to exceed a core temperature $\left(39^{\circ} \mathrm{C}\right)$ and stay within the safety monitoring guidelines. The sweat rate $(\mathrm{L} / \mathrm{h})$ was calculated for each ruck march: ([ $\Delta$ nude body weight $\{\mathrm{kg}\}$ from the start to the finish of ruck march + water consumed during ruck march $\{L\}$ - urine volume during ruck march $\{L\}] / 1$-hour ruck march). ${ }^{27}$ The average and maximum sweat rate over the four ruck marches were determined for each participant. On days 4 and 5 , subjects in the ambient group stayed overnight in the dormitory; however, they performed their regular soldier duties during the day.

Urine collection and analysis. Twenty-one urine samples per soldier were collected at the scheduled 21 time points $(n=$ 567) using pre-labeled specimen cups. Urine samples were analyzed at the U.S. Army Research Institute of Environmental Medicine (USARIEM) Central Laboratory for creatinine, specific gravity, and osmolality (the latter two measurements were not used in the data analyses but were required to monitor potential safety concerns regarding dehydration among participants during the study). Following each study session, 5-mL aliquots were frozen and sent to the CDC laboratory for analysis of three permethrin urinary biomarkers: 3-phenoxybenzoic acid (3-PBA) and cis- and trans-3-(2,2-dichlorovinyl)-2,2-dimethylcyclopropane1-carboxylic acid (DCCA) using a method described in Davis et al. ${ }^{28}$ Limits of detection (LODs) were $0.1 \mu \mathrm{g} / \mathrm{L}$ (3-PBA) and 0.6 $\mu \mathrm{g} / \mathrm{L}$ (trans-DCCA). For cis-DCCA, LODs were $0.6 \mu \mathrm{g} / \mathrm{L}$ for the heat group and $0.5 \mu \mathrm{g} / \mathrm{L}$ for the heat/humidity and ambient groups.

Statistical analysis. Five percent (30/567) of the urine samples had creatinine concentrations outside the range of 20-350 $\mathrm{mg} / \mathrm{dL}$ and were excluded from the statistical analysis $^{29}$ : 15 samples from 10 participants were below $20 \mathrm{mg} / \mathrm{dL}$ creatinine (heat group: three samples, heat/humidity group: 10 samples, and ambient group: two samples) and 15 samples from six participants were above the $350 \mathrm{mg} / \mathrm{dL}$ creatinine cutoff (heat group: 11 samples and ambient group: four samples). Three samples measuring cis-DCCA concentrations recorded values below the $L O D$, and these were replaced with a value of LOD/ $\sqrt{ } 2^{10,30,31}$ (No 3-PBA or trans-DCCA concentrations were measured below the LOD.) The sum of cis- and trans-DCCA ( $\left.\sum D C C A\right)$ was calculated and analyzed in addition to analyzing the DCCA isomers separately.

For descriptive statistics (for demographic, military service, day 1 , and study variables), differences in continuous variables were analyzed using Analyses of Variance (ANOVA) with the Tukey correction to determine the significance of between-group differences. Fisher's exact test was used to calculate differences for categorical variables. Years of active duty service and military occupational specialty (MOS) were included as previous time in the military, and MOS may have had an impact on participants' historical occupational exposure to permethrin.

Using individual $\sum$ DCCA and creatinine concentrations at U12 (12 hours after removing study issued uniform), daily dose estimates $(\mu \mathrm{g} / \mathrm{kg}$-body weight/day) for permethrin uptake were calculated for each subject using a method outlined in Appel et al. ${ }^{12}$ and most recently described in Proctor et al. ${ }^{18}$

To determine differences in permethrin biomarker concentrations between the three study (heat, heat/humidity, and ambient) groups, biomarker concentrations were logtransformed and a linear mixed model was fit using the variance components covariance structure, and exponentiated betas were estimated using a maximum likelihood estimation. Separate models were run for 3-PBA, cis-DCCA, trans-DCCA, and $\sum$ DCCA. In each model, the 10 urine samples (U3-U12) that lie within the absorption curve window (i.e., 12 hours before wearing study-issued uniform to 12 hours after wearing study-issued uniform) were used in the linear mixed models. Participants in the ambient group served as the reference group, and models were adjusted for creatinine (mg/dL) and \% body fat. Total energy expenditure (kcal) over 33 hours, average sweat rate $(\mathrm{L} / \mathrm{h})$, and maximum sweat rate $(\mathrm{L} / \mathrm{h})$ were also examined as possible covariates. Statistical analyses were performed using SAS 9.4 (SAS, Carey, NC).

TABLE 1

Descriptive characteristics of study groups at baseline (day 1)

\begin{tabular}{|c|c|c|c|}
\hline & \multicolumn{3}{|c|}{ Study groups } \\
\hline & \multirow{2}{*}{$\frac{\text { Heat }(n=10)}{\text { Mean }(\mathrm{SD})}$} & \multirow{2}{*}{$\frac{\text { Heat/humidity }(n=10)}{\text { Mean }(\mathrm{SD})}$} & \multirow{2}{*}{$\frac{\text { Ambient }(n=7)}{\text { Mean (SD) }}$} \\
\hline & & & \\
\hline Age (years) & $20.8(3.6)$ & $20.1(1.9)$ & $19.7(3.4)$ \\
\hline Active army service (months) & $6.4(0.7)$ & $6.1(1.1)$ & $7.1(2.4)$ \\
\hline Day 1 BMI (kg/m²) & $24.5(3.1)$ & $23.4(1.7)$ & $23.4(2.3)$ \\
\hline \multirow[t]{2}{*}{ Day $1 \%$ body fat } & $12.3(4.5)$ & $9.8(2.2)$ & $12.9(5.9)$ \\
\hline & $N(\%)$ & $N(\%)$ & $N(\%)$ \\
\hline \multicolumn{4}{|l|}{ Race } \\
\hline Black & $2(20 \%)$ & 0 & $1(14.3 \%)$ \\
\hline White & $8(80 \%)$ & $7(70 \%)$ & $6(85.7 \%)$ \\
\hline Hispanic/Latino & 0 & $2(20 \%)$ & 0 \\
\hline Other & 0 & $1(10 \%)$ & 0 \\
\hline $\begin{array}{l}\text { Army military occupational specialty } \\
(\% \text { infantry })^{\star}\end{array}$ & $10(100 \%)$ & $10(100 \%)$ & $4(57 \%)$ \\
\hline Regular exercise (\% yes) & $8(80 \%)$ & $10(100 \%)$ & $7(100 \%)$ \\
\hline Smoke and/or chew (\% yes)† & $4(40 \%)$ & $2(20 \%)$ & $3(42.9 \%)$ \\
\hline Current alcohol use (\% yes) & $3(30 \%)$ & $6(60 \%)$ & $5(71.4 \%)$ \\
\hline Current caffeine use (\% yes) & $9(90 \%)$ & $10(100 \%)$ & $7(100 \%)$ \\
\hline Ever had a job with pesticides (\%) & $1(10 \%)$ & 0 & $1(14.3 \%)$ \\
\hline Use pesticide around house/garden last & $2(20 \%)$ & 0 & 2 (28.6\%) \\
\hline
\end{tabular}




\section{RESULTS}

Demographic information for the study groups at baseline (day 1) is described in Table 1. A total of 27 male enlisted soldiers wore study-issued permethrin-treated uniforms for 33 consecutive hours in three different environmental conditions. There were no statistically significant differences between the soldiers participating in the three study groups on all background variables other than MOS.

Over the study period, the total energy expenditure over the 33 hours across the three groups is as follows: heat group = 2,077.6 (388.8) kcal, heat/humidity group $=1,940.8$ (451.8) kcal, and ambient group =1,422 (228.8). We observed a significant effect between the study group and total energy expenditure $(F=6.41$, df $=2, P=0.006)$. Differences in energy expenditure were significant between the both the heat and ambient $(P=0.005)$ groups and the heat/humidity and ambient $(P=0.03)$ groups. There were no significant differences in energy expenditure over 33 hours between the heat and heat/ humidity groups. The average sweat rate $(\mathrm{L} / \mathrm{h})$ measured over the four ruck marches was significantly higher in the heat group than the heat/humidity group (heat group $=0.91 \mathrm{~L} / \mathrm{h}$; heat/humidity group $=0.42 \mathrm{~L} / \mathrm{h} ; P<0.0001$ ). A similar result was observed for the maximum sweat rate (heat group $=1.18$ $\mathrm{L} / \mathrm{h}$; heat/humidity group $=0.60 \mathrm{~L} / \mathrm{h} ; P=\leq 0.0001$ ).

The average remaining permethrin concentration on uniform jackets and trousers worn by the heat group was 0.076 (range, $0.069-0.082) \mathrm{mg} / \mathrm{cm}^{2}$ and 0.080 (range, 0.062-0.092) $\mathrm{mg} / \mathrm{cm}^{2}$, respectively. The average permethrin concentration of the uniform jackets worn by the ambient group was 0.090 (range, $0.072-0.12$ ) $\mathrm{mg} / \mathrm{cm}^{2}$ and 0.087 (range, $\left.0.081-0.092\right) \mathrm{mg} / \mathrm{cm}^{2}$ in uniform trousers. The average permethrin concentration measured on worn study uniforms was similar to the unworn uniforms for both jackets $(0.080$ (range, $\left.0.073-0.096) \mathrm{mg} / \mathrm{cm}^{2}\right)$ and trousers $(0.082$ (range, $\left.0.064-0.093) \mathrm{mg} / \mathrm{cm}^{2}\right)$, indicating minimal leaching during the 33-hour wear-time.

Permethrin metabolites 3-PBA and trans-DCCA were detected in $100 \%$ of the urine samples collected regardless of the study group. Cis-DCCA was detected in all but three samples (1 sample each from the heat, heat/humidity, and ambient groups). Figures 2 and 3 provide graphic representation of the geometric mean concentrations of permethrin biomarkers measured in the urine samples taken over the course of the study period (U1-U21). All three study groups had similar biomarker concentrations at baseline; however, an increase in

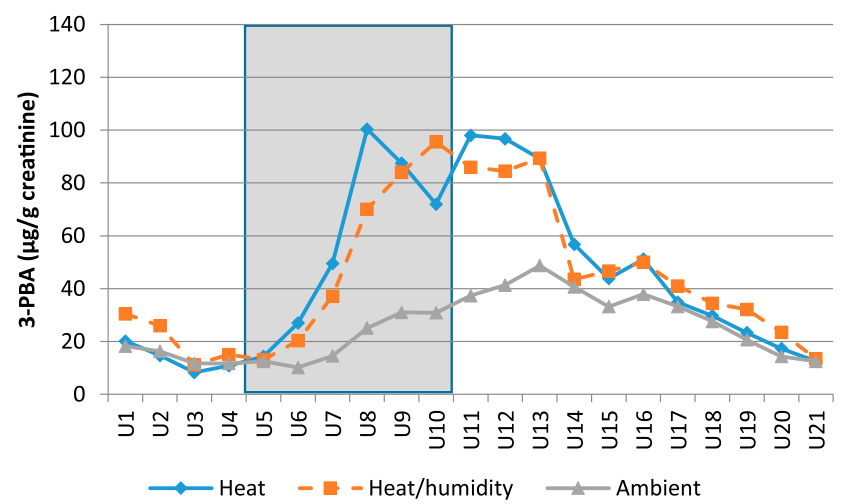

FIGURE 2. Shaded area is the period of time spent under study environmental conditions wearing study-issued uniform. 3-PBA = 3phenoxybenzoic acid. This figure appears in color at www.ajtmh.org.

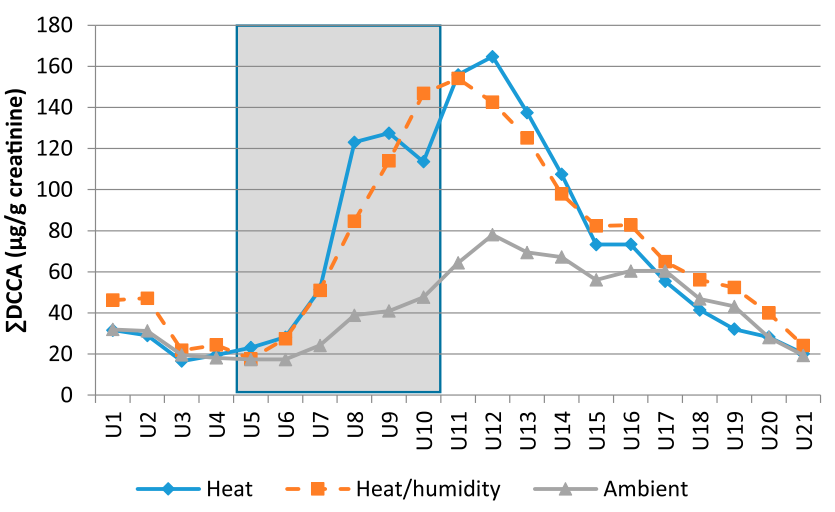

FIGURE 3. Shaded area is the period of time spent under study environmental conditions wearing study-issued uniform $\sum D C C A=$ sum of cis- and trans-DCCA: 3-(2,2-dichlorovinyl)-2,2-dimethylcyclopropane-1-carboxylic acid. This figure appears in color at www.ajtmh.org.

biomarker concentrations was observed in the heat and the heat/humidity groups while participants were wearing the study-issued permethrin-treated uniform (U5 through U10, shaded area) compared with the ambient group. The geometric mean values of DCCA biomarkers continued to increase after the removal of the study-issued uniform (U10). The excretion pattern following the removal of the study uniform was more varied for 3-PBA. All groups returned to baseline concentrations by the final urine sample collection on day 11 (U21).

Median and 95th percentile concentrations for the urine samples used in the repeated mixed models are presented in Table 2.

Despite slight differences in peak excretion, for consistency, daily dose estimates were calculated based on a peak cis- and trans-DCCA excretion at U12 ( 12 hours post-study uniform wear). The average daily dose at U12 was 7.21 (range, 3.31-10.01) $\mu \mathrm{g} / \mathrm{kg} /$ day for the heat group, 6.76 (range, $2.01-10.87) \mu \mathrm{g} / \mathrm{kg} /$ day for the heat/humidity group, and 3.41 (range, 2.62-4.43) $\mu \mathrm{g} / \mathrm{kg} /$ day for the ambient group. A significant effect was observed between the study group and average daily dose $(F=5.53, \mathrm{df}=2, P=0.01)$. Comparing groups, average daily dose was not significantly different between the heat and the heat/humidity groups, but both were significantly higher than the ambient group (heat versus ambient $P$-value $=$ 0.013 ; heat/humidity versus ambient $P$-value $=0.03$ )

Results of the repeated mixed models found significant increases in all permethrin biomarkers in the heat and heat/ humidity groups compared with the ambient group (Table 3). Accounting for repeated measures, 3-PBA concentrations were $90 \%$ higher in soldiers in the heat group than soldiers in the ambient group. Similarly, cis- and trans-DCCA concentrations were $60 \%$ and $80 \%$ higher, respectively. In the heat/ humidity group, concentrations of 3-PBA were $60 \%$ and $50 \%$ higher for cis- and trans-DCCA than the ambient group. No significant differences were observed in permethrin biomarker concentrations between the heat and heat/humidity groups. Creatinine $(\mathrm{mg} / \mathrm{dL})$ was also a significant predictor of urinary concentrations of permethrin biomarkers.

Addition of energy expenditure or the sweat rate as covariates did not have any effect on the pattern of results observed when permethrin biomarker concentrations were used across the study groups (i.e., inclusion did not improve the fit of the model nor were these statistically significant covariates), so these variables were not included in the final models. 
TABLE 2

Creatinine-adjusted median and 95th percentiles $(\mu \mathrm{g} / \mathrm{g}$ creatinine) of permethrin biomarkers (measured for U3-U12) by group

\begin{tabular}{|c|c|c|c|c|}
\hline & 3-PBA & cis-DCCA & trans-DCCA & $\sum \mathrm{DCCA}$ \\
\hline & Median 95th percentile & Median 95th percentile & Median 95th percentile & Median 95th percentile \\
\hline \multicolumn{5}{|c|}{ Heat group $(n=10)$} \\
\hline \multirow[t]{2}{*}{ U3 } & 7.5 & 6.4 & 8.7 & 15.1 \\
\hline & 28.6 & 15.1 & 38.6 & 53.6 \\
\hline \multirow[t]{2}{*}{ U4 } & 9.4 & 5.6 & 11.4 & 17.0 \\
\hline & 35.4 & 17.7 & 37.8 & 52.8 \\
\hline \multirow[t]{2}{*}{ U5 } & 11.3 & 5.7 & 12.7 & 18.5 \\
\hline & 36.3 & 18.7 & 40.2 & 58.9 \\
\hline \multirow[t]{2}{*}{ U6 } & 32.1 & 9.1 & 19.2 & 28.8 \\
\hline & 56.0 & 20.0 & 47.7 & 67.6 \\
\hline \multirow[t]{2}{*}{ U7 } & 53.6 & 14.2 & 43.9 & 58.3 \\
\hline & 89.7 & 29.6 & 67.3 & 83.3 \\
\hline \multirow[t]{2}{*}{ U8 } & 90.8 & 22.9 & 99.0 & 128.3 \\
\hline & 264.9 & 110.7 & 281.6 & 392.3 \\
\hline \multirow[t]{2}{*}{ U9 } & 94.4 & 33.4 & 105.9 & 141.4 \\
\hline & 166.9 & 65.1 & 159.2 & 224.3 \\
\hline \multirow[t]{2}{*}{ U10 } & 61.4 & 28.8 & 83.0 & 109.0 \\
\hline & 178.9 & 74.9 & 168.8 & 243.6 \\
\hline \multirow[t]{2}{*}{ U11 } & 90.4 & 34.4 & 119.1 & 153.1 \\
\hline & 172.0 & 72.1 & 193.9 & 266.1 \\
\hline \multirow[t]{2}{*}{ U12 } & 95.0 & 44.2 & 132.7 & 190.9 \\
\hline & 180.6 & 74.3 & 174.2 & 248.6 \\
\hline \multicolumn{5}{|c|}{ Heat/humidity group $(n=10)$} \\
\hline \multirow[t]{2}{*}{ U3 } & 15.0 & 8.4 & 15.7 & 24.2 \\
\hline & 32.6 & 20.5 & 36.6 & 53.7 \\
\hline \multirow[t]{2}{*}{ U4 } & 13.5 & 7.9 & 13.5 & 20.3 \\
\hline & 41.8 & 19.2 & 45.4 & 64.5 \\
\hline U5 & 14.0 & 8.8 & 12.3 & 20.6 \\
\hline & 38.6 & 15.4 & 39.0 & 54.4 \\
\hline U6 & 18.1 & 8.8 & 15.6 & 25.5 \\
\hline & 84.6 & 42.6 & 91.3 & 133.9 \\
\hline U7 & 38.8 & 14.6 & 30.9 & 48.1 \\
\hline & 62.6 & 35.4 & 63.1 & 95.7 \\
\hline U8 & 71.1 & 19.9 & 51.8 & 73.5 \\
\hline & 118.1 & 51.9 & 132.1 & 167.5 \\
\hline U9 & 82.7 & 27.5 & 82.1 & 109.6 \\
\hline & 146.9 & 54.8 & 173.1 & 206.6 \\
\hline U10 & 93.7 & 44.5 & 108.6 & 153.1 \\
\hline & 166.5 & 89.4 & 204.6 & 292.5 \\
\hline U11 & 86.0 & 47.3 & 131.9 & 185.5 \\
\hline & 139.7 & 81.7 & 185.7 & 243.2 \\
\hline U12 & 91.9 & 43.1 & 107.6 & 150.7 \\
\hline & 151.1 & 94.2 & 176.7 & 270.3 \\
\hline Ambien & & & & \\
\hline U3 & 10.8 & 5.2 & 12.4 & 15.5 \\
\hline & 31.6 & 12.4 & 33.0 & 45.5 \\
\hline U4 & 10.5 & 5.9 & 11.3 & 17.6 \\
\hline & 20.4 & 10.6 & 18.6 & 28.1 \\
\hline U5 & 11.8 & 5.6 & 11.3 & 16.9 \\
\hline & 20.7 & 10.3 & 19.4 & 29.7 \\
\hline U6 & 11.4 & 7.1 & 13.1 & 18.7 \\
\hline & 24.1 & 14.5 & 20.9 & 30.0 \\
\hline U7 & 15.3 & 8.0 & 16.8 & 25.1 \\
\hline & 18.3 & 12.2 & 23.7 & 33.8 \\
\hline U8 & 23.4 & 11.2 & 25.8 & 34.5 \\
\hline & 36.8 & 15.0 & 43.1 & 58.1 \\
\hline U9 & 28.6 & 13.7 & 27.4 & 41.1 \\
\hline & 52.1 & 18.3 & 47.6 & 65.9 \\
\hline U10 & 28.0 & 13.7 & 31.8 & 43.3 \\
\hline & 53.7 & 27.5 & 63.3 & 90.8 \\
\hline U11 & 33.9 & 21.0 & 46.8 & 69.8 \\
\hline & 75.6 & 29.6 & 96.8 & 126.4 \\
\hline U12 & 38.1 & 23.1 & 56.6 & 77.0 \\
\hline & 54.3 & 30.6 & 64.8 & 94.9 \\
\hline
\end{tabular}

U3-U12 = urine samples collected at time points U3 through U12 (from 12 hours before, during, and 12 hours after wearing of treated uniforms). 
TABLE 3

Results from mixed models comparing the effect of heat and heat/humidity on urinary permethrin exposure biomarkers

\begin{tabular}{|c|c|c|c|c|}
\hline & LN(3-PBA) $\beta$ ( $P$-value) & LN(cis-DCCA) $\beta$ (P-value) & LN(trans-DCCA) $\beta$ ( $P$-value) & $\operatorname{LN}\left(\sum\right.$ DCCA $) \beta(P$-value $)$ \\
\hline Intercept & $2.229(<0.0001)$ & $1.621(<0.0001)$ & $2.396(<0.0001)$ & $2.808(<0.0001)$ \\
\hline \multicolumn{5}{|l|}{ Groups } \\
\hline Heat & $0.627(0.001)$ & $0.453(0.005)$ & $0.600(0.001)$ & $0.548(0.001)$ \\
\hline Heat/humidity & $0.497(0.006)$ & $0.398(0.017)$ & $0.410(0.022)$ & $0.395(0.022)$ \\
\hline Ambient & Reference & Reference & Reference & Reference \\
\hline Creatinine $(\mathrm{mg} / \mathrm{dL})$ & $0.006(<0.0001)$ & $0.006(<0.0001)$ & $0.006(<0.0001)$ & $0.006(<0.0001)$ \\
\hline$\%$ Body fat & $0.010(0.51)$ & $0.006(0.65)$ & $0.01(0.49)$ & $0.009(0.54)$ \\
\hline
\end{tabular}

\section{DISCUSSION}

In this study, urinary concentrations of permethrin biomarkers were significantly higher among soldiers wearing permethrin-treated uniforms for 33 hours in the high temperature and combined high temperature and humidity environment (of comparable heat indexes) than soldiers who wore their uniforms in an ambient environment, indicating that these extreme environmental conditions increase absorption of permethrin. We observed no significant differences between the heat and heat/humidity groups in either permethrin biomarkers concentrations or the average daily dose of permethrin.

Soldiers in the heat and heat/humidity groups showed increasing biomarker concentrations shortly after entering the simulated environmental conditions, and then biomarker concentrations decreased back to baseline levels only after leaving the simulated environmental conditions. Although the permethrin biomarker concentrations measured in this study were well above U.S. national values, ${ }^{10}$ the observed significant increases in permethrin biomarker concentrations among the heat group and the heat/humidity group remained on average 3-3.5 times below the WHO ADI, assuming $50 \%$ absorption of oral dose $(25 \mu \mathrm{g} / \mathrm{kg} / \mathrm{day})$, with the maximum average calculated daily dose in the heat/humidity group ( $10.87 \mu \mathrm{g} / \mathrm{kg} / \mathrm{day})$ being almost two times lower. Although the 33-hour wear time scenario implemented in this study was meant to reflect a sustained training or operational period, it is possible that the maximum permethrin absorption and excretion levels were not reached during the study period and the biomarker concentrations could have increased further with more time in the hot and/or humid environments.

Energy expenditure has been demonstrated to impact permethrin exposure in highly active soldiers wearing new permethrin-treated uniforms in training $\left(3,400-3,700\right.$ kcals $\left.^{17}\right)$ but was not observed to be associated with permethrin exposure in a study with Army National Guard Soldiers, ${ }^{32}$ with energy expenditure rates averaging $\sim 3,000 \mathrm{Kcal} /$ day. In this study, across all groups, the energy expenditure was even lower (1,400-2,000 kcals over 33 hours) and, therefore, may not be high enough to meet a threshold where it might impact permethrin exposure. It is possible that the differential energy expenditure levels between groups may have played a role in differential permethrin exposure, but the analyses to examine this did not support this.

These results support the findings of higher concentrations of permethrin biomarkers among a group of forestry workers. ${ }^{16}$ When the workers performed a minimum of 4 hours in hot and humid conditions $\left(\sim 25^{\circ} \mathrm{C}, 60 \%\right.$ relative humidity [rh]), peak permethrin excretion increased compared with peak excretion measured after working in comfort conditions (20-22 $\left.{ }^{\circ} \mathrm{C}, 45-50 \% \mathrm{rh}\right)$. In addition, further increases in peak permethrin metabolite levels were noted when an additional physical workload component was added to the hot and humid conditions, but a weak correlation was observed between sweat and excretion of permethrin metabolites. Although consistent with our findings, the biomarker concentrations measured in Rossbach et al. ${ }^{16}$ are not directly comparable with those observed in our study population because spot urine samples were collected at a single time point, after 4 hour of work, and unadjusted biomarker concentrations were presented. Few other occupational studies have examined the effect of elevated temperature and humidity on permethrin exposure among other outdoor workers. Biomonitoring studies of male and female farmworkers and pest control workers have all found evidence of occupational exposure to permethrin. ${ }^{33-37}$ If the work described in these studies was conducted in high-temperature or hot/humid conditions, it is reasonable to assume that based on the present findings, permethrin absorption would also be elevated.

Permethrin excretion patterns described in this study were captured under both hot/humid and ambient environmental conditions. However, there are some limitations on the generalizability of our findings to individuals working under such conditions. First, although the study design did not exclude female volunteers, we did not have any female participants and, thus, were not able to examine whether elevated temperature and humidity affects female soldiers to the same level. However, in another occupational cohort, Latina female farmworkers and non-farmworkers had urinary concentrations of 3-PBA that were similar to their male counterparts. $^{34}$ Second, most of our participants were infantry soldiers with relatively low \% body fat (with minimal variability), a factor that have been positively associated with permethrin biomarker concentrations in other studies ${ }^{8,17}$ but not in this study.

Permethrin-treated army uniforms are a highly effective personal protective measure against insect bites and contracting vector-borne diseases. The CDC recently reported that the number of documented cases of vector-borne diseases reported in the United States has more than doubled from 2004 (> 22,000) to 2016 (> 48,000), with an emergence of nine newly reported vector-borne illnesses during that same time period. ${ }^{38}$ As the long-term use of these uniforms continues to be a vital part of protecting soldiers from diseasecarrying insects, it is important to examine factors that affect permethrin exposure and absorption and, thus, the conditions that contribute to higher dose. The findings of this study suggest that permethrin exposure increases significantly 
while wearing a permethrin-treated uniform in extreme environments (heat and combined heat/humidity) compared with ambient environmental conditions.

Received July 22, 2019. Accepted for publication February 22, 2020. Published online March 30, 2020.

Acknowledgments: We acknowledge the contribution of additional USARIEM study team members who assisted in the data collection for this project and the laboratory technicians at the USARIEM Central Laboratory who performed sample analyses. We also greatly appreciate the expertise and input from Melynda Perry and Amy Johnson (Textile Materials Evaluation Team, Combat Capabilities Development Command Soldier Center, Natick, MA) who consulted on the project and conducted the permethrin analyses of the uniforms, respectively. We thank Mark Davis and Isuru Vidanage at the Centers for Disease Control and Prevention for technical assistance in the quantification of the pyrethroid biomarkers in urine.

Financial support: This research was supported through intramural core funding from the U.S. Army Medical Research and Development Command Military Operational Medicine Research Program to USARIEM.

Disclaimer: The views expressed are those of the authors and do not reflect the official policy of the Department of the Army or the Department of Defense. The findings and conclusions in this report are those of the authors and do not necessarily represent the official position of the CDC. The use of trade names is for identification only and does not imply endorsement by the CDC, the Public Health Service, or the U.S. Department of Health and Human Services.

Authors' addresses: Alexis L. Maule, Cherokee Nation Strategic Programs, Tulsa, OK, and Defense Health Agency - U.S. Army Satellite, Aberdeen Proving Ground, MD, E-mail: alexis.I.maule.ctr@mail.mil. Kristin J. Heaton, Kathryn M. Taylor, Katelyn I. Guerriere, Caitlin C. Haven, and Susan P. Proctor, Military Performance Division, United States Army Research Institute of Environmental Medicine, Natick, MA, E-mails: kristin.j.heaton.civ@mail.mil, kathryn.m.taylor41.civ@mail.mil, katelyn.i.guerriere.civ@mail.mil, caitlin.c.haven.civ@mail.mil, and susan. p.proctor.civ@mail.mil. Bruce Cadarette and Robert W. Kenefick, Thermal and Mountain Medicine Division, United States Army Research Institute of Environmental Medicine, Natick, MA, E-mails: bruce.s.cadarette.civ@mail.mil and bobwk@comcast.net. Matthew M. Scarpaci, Hassenfeld Child Health Innovation Institute, Brown University, Providence, RI, E-mail: scarpacm@bu.edu. Maria Ospina and Antonia M. Calafat, Division of Laboratory Sciences, Centers for Disease Control and Prevention, National Center for Environmental Health, Atlanta, GA, E-mails: meo3@cdc.gov and aic7@cdc.gov.

\section{REFERENCES}

1. United States Army, 2012. All Army Activities (ALARACT) 289/ 2012, October 16, 12, Headquarters Department of Army. Subject: Factory Treated Army Combat Uniforms with Permethrin (Acu Permethrin).

2. Banks SD, Murray N, Wilder-Smith A, Logan JG, 2014. Insecticide-treated clothes for the control of vector-borne diseases: a review on effectiveness and safety. Med Vet Entomol 28 (Suppl 1): 14-25.

3. Londono-Renteria B et al., 2015. Long-lasting permethrinimpregnated clothing protects against mosquito bites in outdoor workers. Am J Trop Med Hyg 93: 869-874.

4. Most B, Pommier de Santi V, Pagès F, Mura M, Uedelhoven WM, Faulde MK, 2017. Long-lasting permethrin-impregnated clothing: protective efficacy against malaria in hyperendemic foci, and laundering, wearing, and weathering effects on residual bioactivity after worst-case use in the rain forests of French Guiana. Parasitol Res 116: 677-684.

5. Faulde MK, Rutenfranz M, Keth A, Hepke J, Rogge M, Görner A, 2015. Pilot study assessing the effectiveness of factory-treated, long-lasting permethrin-impregnated clothing for the prevention of tick bites during occupational tick exposure in highly infested military training areas, Germany. Parasitol Res 114: 671-678.
6. Richards SL, Balanay JAG, Harris JW, 2015. Effectiveness of permethrin-treated clothing to prevent tick exposure in foresters in the central Appalachian region of the USA. Int J Environ Health Res 25: 453-462.

7. Sullivan KM et al., 2019. Bioabsorption and effectiveness of longlasting permethrin-treated uniforms over three months among North Carolina outdoor workers. Parasit Vectors 12: 52.

8. Proctor SP, Maule AL, Heaton KJ, Adam GE, 2014. Permethrin exposure from fabric-treated military uniforms under different wear-time scenarios. J Exp Sci Environ Epidemiol 24: 572-578.

9. Maule AL, Scarpaci MM, Proctor SP, 2019. Urinary concentrations of permethrin metabolites in US army personnel in comparison with the US adult population, occupationally exposed cohorts, and other general populations. Int $J$ Hyg Environ Health 222: 355-363.

10. Center for Disease Control and Prevention (CDC), 2019. Fourth National Report on Human Exposure to Environmental Chemicals, Updated Tables, January: Volume 1. 2019. Available at: https://www.cdc.gov/exposurereport/pdf/FourthReport_Updated Tables_Volume1_Jan2019-508.pdf. Accessed June 6, 2019.

11. United States Environmental Protection Agency (USEPA), 2007. Reregistration Eligibility Decision (RED) for Permethrin. Updated December 2007. Available at: https://archive.epa.gov/ pesticides/reregistration/web/pdf/permethrin_amended_red. pdf. Accessed January 18, 2018.

12. Appel KE, Gundert-Remy U, Fischer H, Faulde M, Mross KG, Letzel S, Rossbach B, 2008. Risk assessment of Bundeswehr (German Federal Armed Forces) permethrin-impregnated battle dress uniforms (BDU). Int $J$ Hyg Environ Health 211: 88-104.

13. Rossbach B, Appel KE, Mross KG, Letzel S, 2010. Uptake of permethrin from impregnated clothing. Toxicol Lett 192: 50-55.

14. Rossbach B, Kegel $P$, Süß H, Letzel S, 2016. Biomonitoring and evaluation of permethrin uptake in forestry workers using permethrin-treated tick-proof pants. J Expo Sci Epidemiol 26: 95-103.

15. Kegel P, Letzel S, Rossbach B, 2014. Biomonitoring in wearers of permethrin impregnated battle dress uniforms in Afghanistan and Germany. Occup Environ Med 71: 112-117.

16. Rossbach B, Niemietz A, Kegel P, Letzel S, 2014. Uptake and elimination of permethrin related to the use of permethrin treated clothing for forestry workers. Toxicol Lett 231: 147-153.

17. Proctor SP, Scarpaci MM, Maule AL, Heaton KJ, Taylor K, Haven CC, Rood J, Ospina M, Calafat AM, 2018. Role of body composition and physical activity on permethrin urinary biomarker concentrations while wearing treated military uniforms. Toxicol Lett 299: 210-217.

18. Proctor SP, Maule AL, Heaton KJ, Cadarette BS, Guerriere KI, Haven CC, Taylor KM, Scarpaci MM, Ospina M, Calafat AM, 2019. Permethrin exposure from wearing fabric-treated military uniforms in high heat conditions under varying wear-time scenarios. J Expo Sci Environ Epidemiol. epub date February 6, 2019. doi: 10.1038/s41370-019-0120-y.

19. Gordon CJ, 2003. Role of environmental stress in the physiological response to chemical toxicants. Environ Res 92: 1-7.

20. Anderson SE, Meade BJ, 2014. Potential health effects associated with dermal exposure to occupational chemicals. Environ Health Insights. 8 (Suppl 1): 51-62.

21. MacFarlane E, Carey R, Keegel T, El-Zaemay S, Fritschi L, 2013. Dermal exposure associated with occupational end use of pesticides and the role of protective measures. SafHealth Work 4: $136-141$.

22. Poet TS, McDougal JN, 2002. Skin absorption and human risk assessment. Chem Biol Interact 140: 19-34.

23. Baty International, 2010. The Harpenden Skinfold Caliper HSB-BI. West Sussex, United Kingdom: Baty International Ltd.

24. Headquarters, Department of the Army, 2009. Revision A. Coat, Army Combat Uniform. Washington, DC: Headquarters, Department of the Army. June 10, 2009. Military Specification: GL PD 07-13A.

25. Headquarters, Department of the Army, 2009. Revision A. Trousers, Army Combat Uniform. Washington, DC: Headquarters, 
Department of the Army. June 10, 2009. Military Specification: GL/PD 07-14A.

26. Heil DP, 2006. Predicting activity energy expenditure using the Actical activity monitor. Res Q Exerc Sport 77: 64-80.

27. Sawka MN, Burke LM, Eichner ER, Maughan RJ, Montain SJ, Stachenfeld NS, 2007. American College of Sport Medicine position stand. Exercise and fluid replacement. Med Sci Sports Exerc 39: 377-390.

28. Davis MD, Wade EL, Restrepo PR, Roman-Esteva W, Bravo R, Kuklenyik P, Calafat AM, 2013. Semi-automated solid phase extraction method for the mass spectrometric quantification of 12 specific metabolites of organophosphorus pesticides, synthetic pyrethroids, and select herbicides in human urine. J Chromatogr B Analyt Technol Biomed Life Sci 929: 18-26.

29. World Health Organization, 1996. Biological Monitoring of Chemical Exposure in the Workplace Guidelines, Vol. 1. Geneva, Switzerland: World Health Organization.

30. Hornung RW, Reed LD, 1990. Estimation of average concentration in the presence of nondetectable values. Appl Occup Environ Hyg 5: 46-51.

31. U.S. EPA, 2006, Data Quality Assessment: Statistical Methods for Practitioners. EPA QA/G-9S, 131. Available at: https:// www.epa.gov/sites/production/files/2015-08/documents/g9sfinal.pdf. Accessed September 19, 2019.

32. Scarpaci MM, Haven CC, Maule AL, Heaton KJ, Taylor KM, Rood J, Ospina M, Calafat AM, Proctor SP, 2020. The effect of body composition and energy expenditure on permethrin biomarker concentrations among US army national guard members. J Occup Environ Med 62: 210-216.

33. Arcury TA, Chen H, Laurienti PJ, Howard TD, Barr DB, Mora DC, Quandt SA, 2018. Farmworker and non-farmworker Latino immigrant men in North Carolina have high levels of specific pesticide urinary metabolites. Arch Environ Occup Health 73: 219-227.

34. Arcury TA, Laurienti PJ, Talton JW, Chen H, Howard TD, Barr DB, Mora DC, Quandt SA, 2018. Pesticide urinary metabolites among Latina farmworkers and nonfarmworkers in North Carolina. J Occup Environ Med 60: e63-e71.

35. Hardt J, Angerer J, 2003. Biological monitoring of workers after the application of insecticidal pyrethroids. Int Arch Occup Environ Health 76: 492-498.

36. Wang $D$ et al., 2007. Biological monitoring of pyrethroid exposure of pest control workers in Japan. J Occup Health 49: 509-514.

37. Ferland S, Côte J, Ratelle M, Thuot R, Bouchard M, 2015. Detailed urinary excretion time courses of biomarkers of exposure to permethrin and estimated exposure in workers of a corn production farm in Quebec, Canada. Ann Occup Hyg 59: 1152-1167.

38. Rosenberg R et al., 2018. Vital signs: trends in reported vectorborne disease cases - United States and territories, 2004-2016. MMWR Morb Mortal Wkly Rep 67: 496-501. 